\title{
Complexity of a Discrete-Time Predator-Prey Model Involving Prey Refuge Proportional to Predator
}

\author{
P.K. Santra ${ }^{1}$, Hasan S. Panigoro ${ }^{2 *}$, G.S. Mahapatra ${ }^{3}$ \\ ${ }^{1}$ Maulana Abul Kalam Azad University of Technology, Kolkata-700064, India \\ ${ }^{2}$ Department of Mathematics, State University of Gorontalo, Bone Bolango 96128, Indonesia \\ ${ }^{3}$ National Institute of Technology Puducherry, Karaikal-609609, India \\ *Corresponding author. Email: hspanigoro@ung.ac.id
}

ABSTRACT

In this paper, a discrete-time predator-prey model involving prey refuge proportional to predator density is studied. It is assumed that the rate at which prey moves to the refuge is proportional to the predator density. The fixed points, their local stability, and the existence of Neimark-Sacker bifurcation are investigated. At last, the numerical simulations consisting of bifurcation diagrams, phase portraits, and time-series are given to support analytical findings. The occurrence of chaotic solutions are also presented by showing the Lyapunov exponent while some parameters are varied.

Keywords:

Chaos; Neimark-Sacker Bifurcation; Predator-Prey; Refuge; Stability

How to Cite:

P.K. Santra, H.S. Panigoro, and G.S. Mahapatra, "Complexity of a Discrete predator-prey model Incorporating Prey Refuge Proportional to Predator", Jambura J. Math., vol. 4, No. 1, pp. 50-63, 2022, doi: https://doi.org/10.34312/jjom.v4i1.11918

\section{Introduction}

The interaction between prey and predator is the most important process in maintaining the balance of the ecosystem. Several investigators studied the predator-prey model in the continuous-time domain. Depending on time, we can divide predator-prey model into two types, discrete domain system [1-9] and continuous domain system [10-15]. The discrete-time predator-prey model has reached dynamics, and interesting qualitative behavior for the study incorporating refuge, different functional responses, harvesting, delays, etc.

The predator-prey model is a substantial concept in different quarters of Ecology. Species are fighting continuously with each other for food and space with an existing relation among different species. Some degree of protection is naturally preserved to a certain amount of prey populations by establishing some safe places called refuges [6, 16-18]. The safe places are preserved in nature to reduce the predation rate and increase the life opportunity of prey.

If we consider the way to model the predator-prey interaction, the differential equation is used as the operator for the continuous domain and the difference equation for the 
discrete domain. The differential equation is usually used for populations with overlapping generations where the birth process occurs continuously. For example, some models use first-order derivative $[16,19]$ and also we can find the model with fractional-order derivative $[14,15,20]$. Some organisms have regular breeding seasons for their births such as monocarpic plants and semelparous animals. Thus, the discrete approaches are regarded as more suitable for these cases. The predator-prey interactions with discrete-time models show the complexity of dynamical behavior rather than the continuous ones [1-3].

Ruxton [21] presented a different term refuge to investigate the stability of predator-prey models. Cressman and Garay [22] discussed the evolutionary stability of the ecosystem of the predator-prey refuge model. Tao et.al [18] presented the effect of prey refuge and harvesting on the dynamics of a predator-prey model with a generalized functional response. Many comprehensive reseaches have been carried out by scientists in involving refuge to predator-prey interaction [10,11, 18, 21-29]. Most of the literature on the predator-prey model considered refuge as a constant amount of prey species or proportional to prey density incorporating through a different form of functional responses $[2,4-6,10,11,18,21-29]$.

In our works, the different points of view in expressing the prey refuge term are considered. We assume that the prey refuge is proportional to predator density. The earlier research works presented that the use of refuges by a fraction of prey, or the constant number of prey exerts a stabilizing effect in the dynamics of the interacting populations. For now, we examine those pronouncements which assume that the quantity of prey in refugia is proportional to predators, and we analyze the dynamic properties of such a predator-prey model with prey self-limitation.

We present this article as follows. In Section 2, the model formulation of a discrete-time predator-prey model with refuge is given. Section 3 deals with results and discussion consist of the fixed points, their existence conditions, their local stability, the occurrence of Neimark-Sacker bifurcation, the chaos control, and some numerical simulations. We end our works with conclusion in Section 4.

\section{Model Formulation}

In this section, the mathematical modeling is presented. The model is constructed based on the gause-type predator-prey model where the prey growth logistically and hunting by predator for foods. The generalized form is given by

$$
\begin{aligned}
& \frac{d x}{d t}=r x\left(1-\frac{x}{k}\right)-c \varphi(x) y \\
& \frac{d y}{d t}=d \varphi(x) y-f y
\end{aligned}
$$

where variables and parameters given by Table 1 . Denote that $\varphi(0)=0, \varphi^{\prime}(x)>0$ for $x>0$. There is a quantity $x_{r}$ of prey population which incorporates refuges for the functional responses $\varphi(x)$ replaced by $\varphi\left(x-x_{r}\right)$, then incorporating prey refuges considering $\varphi(x)=x$ and $x_{r}=b y$. The discrete-time is obtained by the discretization scheme as follows: $\frac{d x}{d t}=\frac{x_{t+h}-x_{t}}{h}, \frac{d y}{d t}=\frac{y_{t+h}-y_{t}}{h}$, where $x_{t}$ and $y_{t}$ are respectively the 
Complexity of a Discrete predator-prey model Incorporating Prey Refuge...

Table 1. Variables and parameters biological descriptions for model (3)

\begin{tabular}{cl}
\hline Variables and parameters & Biological description \\
\hline$x$ & The density of prey \\
$t$ & The density of predator \\
$r$ & Time \\
$k$ & The intrinsic growth rate of prey \\
$c$ & The environmental carrying capacity of prey \\
$d$ & The predation rate \\
& The efficiency with which predator convert consumed \\
& prey into new predator \\
\end{tabular}

prey and predator populations densities at time $t$. For $(n+1)$ th generation, $f=1, h \rightarrow 1$, and replacing $t$ by $n$, we obtain

$$
\begin{aligned}
& x_{n+1}=(r+1) x_{n}\left(1-\frac{r}{k(r+1)} x_{n}\right)-c\left(x_{n}-b y_{n}\right) y_{n} \\
& y_{n+1}=d\left(x_{n}-b y_{n}\right) y_{n}
\end{aligned}
$$

To reduce the parameters of the model, we use the rescale $\frac{r}{k(r+1)}=1$ and $(r+1)=a$. Therefore, the following simplified discrete-time model is obtained.

$$
\begin{aligned}
& x_{n+1}=a x_{n}\left(1-x_{n}\right)-c\left(x_{n}-b y_{n}\right) y_{n} \\
& y_{n+1}=d\left(x_{n}-b y_{n}\right) y_{n}
\end{aligned}
$$

where are all of the biological parameters $a, b, c$, and $d$ are positive.

\section{Results and Discussion}

\subsection{Dynamical Analysis of Proposed Discrete-Time Predator-Prey Model}

The fixed points of the model (3) are obtained by investigating the positive solutions of the following equations.

$$
\begin{aligned}
& x=a x(1-x)-c(x-b y) y \\
& y=d(x-b y) y
\end{aligned}
$$

Hence, three fixed points are achieved as follows.

1. The origin $P_{0}=(0,0)$ which always exists,

2. The axial point $P_{1}=\left(\frac{a-1}{a}, 0\right)$, which exists if $a>1$,

3. The interior point $P_{2}=\left(x_{2}, y_{2}\right)$ which exists if $x_{2}$ is positive solution of the quadratic equation $x^{2}+A x+B=0, A=\frac{1-a}{a}+\frac{c}{a b d}, B=-\frac{c}{a b d^{2}}, y_{2}=\frac{x_{2}}{b}-\frac{1}{b d}$, and $x_{2}>\frac{b}{b d}$.

The fixed points $P_{i}, i=0,1,2$ are respectively represent both populations extinction point, predator extinction point, and co-existence point. 
Now, the local dynamics for each fixed point is discussed. Using linearization, the following Jacobian matrix at $(x, y)$ given by

$$
J=\left[\begin{array}{cc}
a(1-2 x)-c y & -c(x-2 b y) \\
d y & d(x-2 b y)
\end{array}\right],
$$

which gives characteristic equation: $\lambda^{2}-\operatorname{Tr}(J) \lambda+\operatorname{Det}(J)=0$, where

$$
\begin{aligned}
\operatorname{Tr}(J) & =a+x(d-2 a)-y(c+2 b d)(\text { Trace of } J), \\
\operatorname{Det}(J) & =a d(1-2 x)(x-2 b y)(\text { Determinant of } J) .
\end{aligned}
$$

Hence, model (3) is said to be

(i) a dissipative dynamical system if $|a d(1-2 x)(x-2 b y)|<1$, and

(ii) a conservative dynamical one if and only if $|a d(1-2 x)(x-2 b y)|=1$.

Furthermore, the stability of each fixed point is given by the following theorem.

Theorem 1. The origin $P_{0}=(0,0)$ is sink if $0<a<1$, saddle if $a>1$, and non hyperbolic if $a=1$.

proof. At $P_{0}$, we have the Jacobian matrix $J_{(x, y)=(0,0)}=\left[\begin{array}{ll}a & 0 \\ 0 & 0\end{array}\right]$, which gives eigenvalues $\lambda_{1}=a$ and $\lambda_{2}=0$. Since $\left|\lambda_{1}\right|=0<1$, the stability depends on $\lambda_{1}$. It is clear that $\left|\lambda_{1}\right|=|a|=a$. Following Lemma 1 in [7], we have Theorem 1 .

Theorem 2. The axial point $P_{1}=\left(\frac{a-1}{a}, 0\right)$ is

(i) a sink if $|2-a|<1$ and $\left|\frac{d(a-1)}{a}\right|<1$,

(ii) a source if $|2-a|>1$ and $\left|\frac{d(a-1)}{a}\right|>1$,

(iii) a saddle if $|2-a|>1$ and $\left|\frac{d(a-1)}{a}\right|<1$ or $|2-a|<1$ and $\left|\frac{d(a-1)}{a}\right|>1$, and

(iv) a non hyperbolic if $|2-a|=1$ or $\left|\frac{d(a-1)}{a}\right|=1$.

proof. In this fixed point, we have $J_{(x, y)=\left(\frac{a-1}{a}, 0\right)}=\left[\begin{array}{cc}2-a & -\frac{c(a-1)}{a} \\ 0 & \frac{d(a-1)}{a}\end{array}\right]$, and hence the eigenvalues are $\lambda_{1}=2-a$ and $\lambda_{2}=\frac{d(a-1)}{a}$. Thus, we obtain Theorem 2 by obeying Lemma 1 in [7].

Theorem 3. The local stability of the interior point $P_{2}=\left(x_{2}, y_{2}\right)$ satisfies one of the following statements.

(i) a sink if $1+\operatorname{Tr}(\hat{J})+\operatorname{Det}(\hat{J})>0$ and $\operatorname{Det}(\hat{J})<1$,

(ii) a source if $1+\operatorname{Tr}(\hat{J})+\operatorname{Det}(\hat{J})>0$ and $\operatorname{Det}(\hat{J})>1$,

(iii) a saddle if $1+\operatorname{Tr}(\hat{J})+\operatorname{Det}(\hat{J})<0$, and

(iv) a non hyperbolic if $1+\operatorname{Tr}(\hat{J})+\operatorname{Det}(\hat{J})=0$ and $\operatorname{Tr}(\hat{J}) \neq 0,2$, or $[\operatorname{Tr}(\hat{J})]^{2}-4 \operatorname{Det}(\hat{J})<$ 0 and $\operatorname{Det}(\hat{J})=1$, 
where $\operatorname{Tr}(\hat{J})=a+x_{2}(d-2 a)-y_{2}(c+2 b d)$ and $\operatorname{Det}(\hat{J})=a d\left(1-2 x_{2}\right)\left(x_{2}-2 b y_{2}\right)$.

proof. Let $F(\lambda)=\lambda^{2}-\lambda \operatorname{Tr}(\hat{J})+\operatorname{Det}(\hat{J})$. Thus, we get

$$
\begin{aligned}
F(1) & =1-\operatorname{Tr}(\hat{J})+\operatorname{Det}(\hat{J}) \\
& =1-\left[a+x_{2}(d-2 a)-y_{2}(c+2 b d)\right]+\left[a d\left(1-2 x_{2}\right)\left(x_{2}-2 b y_{2}\right)\right], \\
f(-1) & =1+\operatorname{Tr}(\hat{J})+\operatorname{Det}(\hat{J}) \\
& =1+\left[a+x_{2}(d-2 a)-y_{2}(c+2 b d)\right]+\left[a d\left(1-2 x_{2}\right)\left(x_{2}-2 b y_{2}\right)\right] .
\end{aligned}
$$

By applying Lemma 2 in [7], the given stability conditions hold.

At the interior fixed point $P_{2}\left(x_{2}, y_{2}\right)$, if $1-\operatorname{Tr}(\hat{J})+\operatorname{Det}(\hat{J})>0,1+\operatorname{Tr}(\hat{J})+\operatorname{Det}(\hat{J})=0$, and $\operatorname{Tr}(\hat{J}) \neq 0,2$ then at $\left(x_{2}, y_{2}\right)$ the model can undergo flip bifurcation.

Again at $P_{2}\left(x_{2}, y_{2}\right)$, if $1-\operatorname{Tr}(\hat{J})+\operatorname{Det}(\hat{J})>0,(\operatorname{Tr}(\hat{J}))^{2}-4 \operatorname{Det}(\hat{J})<0$ and $\operatorname{Det}(\hat{J})=1$ then at $\left(x_{2}, y_{2}\right)$, the model can undergo Neimark-Sacker bifurcation.

\subsection{Neimark-Sacker Bifurcation of Proposed Model}

Neimark-Sacker bifurcation occurs when a fixed point losses its stability and nearby solutions are isolated by an invariant closed curve simultaneously when a parameter is varied. In this subsection, we present the occurrence of Neimark-Sacker bifurcation around the interior point $P_{2}$ of model (3) driven by a parameter in the following set.

$$
A=\left\{(a, b, c, d): 1-\operatorname{Tr}(\hat{J})+\operatorname{Det}(\hat{J})>0,(\operatorname{Tr}(\hat{J}))^{2}-4 \operatorname{Det}(\hat{J})<0, \operatorname{Det}(\hat{J})=1\right\}
$$

We analyze the existence of Neimark-Sacker bifurcation by set the parameter $b$ as the bifurcation parameter. Further $b^{*}\left(\left|b^{*}\right| \lll 1\right)$ is the perturbation of $b$, we consider a perturbation of the model as follows:

$$
\begin{aligned}
& x_{n+1}=a x_{n}\left(1-x_{n}\right)-c\left(x_{n}-\left(b+b^{*}\right) y_{n}\right) y_{n} \equiv f\left(x_{n}, y_{n}, b^{*}\right) \\
& y_{n+1}=d\left(x_{n}-\left(b+b^{*}\right) y_{n}\right) y_{n} \equiv g\left(x_{n}, y_{n}, b^{*}\right)
\end{aligned}
$$

Now, we transform the fixed point $P_{2}\left(x_{2}, y_{2}\right)$ into the origin by using equations $u_{n}=$ $x_{n}-x_{2}$ and $v_{n}=y_{n}-y_{2}$ simultaneously with applying a Taylor series at $\left(u_{n}, v_{n}\right)=(0,0)$ to the model (6). Hence, we obtain

$$
\begin{aligned}
u_{n+1}= & \alpha_{1} u_{n}+\alpha_{2} v_{n}+\alpha_{11} u_{n}^{2}+\alpha_{12} u_{n} v_{n}+\alpha_{22} v_{n}^{2}+\alpha_{111} u_{n}^{3} \\
& +\alpha_{112} u_{n}^{2} v_{n}+\alpha_{122} u_{n} v_{n}^{2}+\alpha_{222} v_{n}^{3}+O\left(\left(\left|u_{n}\right|+\left|v_{n}\right|\right)^{4}\right) \\
v_{n+1}= & \beta_{1} u_{n}+\beta_{2} v_{n}+\beta_{11} u_{n}^{2}+\beta_{12} u_{n} v_{n}+\beta_{22} v_{n}^{2}+\beta_{111} u_{n}^{3} \\
& +\beta_{112} u_{n}^{2} v_{n}+\beta_{122} u_{n} v_{n}^{2}+\beta_{222} v_{n}^{3}+O\left(\left(\left|u_{n}\right|+\left|v_{n}\right|\right)^{4}\right)
\end{aligned}
$$

Where $\alpha_{1}=f_{x}\left(x_{2}, y_{2}, 0\right)=a\left(1-2 x_{2}\right)-c y_{2}, \alpha_{2}=f_{y}\left(x_{2}, y_{2}, 0\right)=-c\left(x_{2}-2 b y_{2}\right)$, $\alpha_{11}=f_{x x}\left(x_{2}, y_{2}, 0\right)=-2 a, \alpha_{12}=f_{x y}\left(x_{2}, y_{2}, 0\right)=-c, \alpha_{22}=f_{y y}\left(x_{2}, y_{2}, 0\right)=2 b c$, $\alpha_{111}=f_{x x x}\left(x_{2}, y_{2}, 0\right)=0, \alpha_{112}=f_{x x y}\left(x_{2}, y_{2}, 0\right)=0, \alpha_{122}=f_{x y y}\left(x_{2}, y_{2}, 0\right)=0$, $\alpha_{222}=f_{y y y}\left(x_{2}, y_{2}, 0\right)=0, \beta_{1}=g_{x}\left(x_{2}, y_{2}, 0\right)=d y_{2}, \beta_{2}=g_{y}\left(x_{2}, y_{2}, 0\right)=d\left(x_{2}-2 b y_{2}\right)$, $\beta_{11}=g_{x x}\left(x_{2}, y_{2}, 0\right)=0, \beta_{12}=g_{x y}\left(x_{2}, y_{2}, 0\right)=d, \beta_{22}=g_{y y}\left(x_{2}, y_{2}, 0\right)=-2 b d$, $\beta_{111}=g_{x x x}\left(x_{2}, y_{2}, 0\right)=0, \beta_{112}=g_{x x y}\left(x_{2}, y_{2}, 0\right)=0, \beta_{122}=g_{x y y}\left(x_{2}, y_{2}, 0\right)=0$, $\beta_{222}=g_{y y y}\left(x_{2}, y_{2}, 0\right)=0$. 
If we compute the Jacobian matrix of model $(7)$ at $\left(u_{n}, v_{n}\right)=(0,0)$, the following characteristic equation and eigenvalues are obtained.

$$
\begin{array}{r}
\lambda^{2}-\operatorname{Tr}\left(J_{1}\left(b^{*}\right)\right) \lambda+\operatorname{Det}\left(J_{1}\left(b^{*}\right)\right)=0 \\
\lambda_{1,2}\left(b^{*}\right)=\frac{\operatorname{Tr}\left(J_{1}\left(b^{*}\right)\right) \pm i \sqrt{4 \operatorname{Det}\left(J_{1}\left(b^{*}\right)\right)-\left(\operatorname{Tr}\left(J_{1}\left(b^{*}\right)\right)\right)^{2}}}{2} .
\end{array}
$$

From $\left|\lambda_{1,2}\left(b^{*}\right)\right|=1$, when $b^{*}=0$ we have $\left|\lambda_{1,2}\left(b^{*}\right)\right|=\left[\operatorname{Det}\left(J_{1}\left(b^{*}\right)\right)\right]^{\frac{1}{2}}$ and $l=\left[\frac{d\left|\lambda_{1,2}\left(b^{*}\right)\right|}{d b^{*}}\right]_{h^{*}=0} \neq 0$. In addition to that when $b^{*}=0, \lambda_{1,2}^{i} \neq 1, i=1,2,3,4$, which is equivalent to $\operatorname{Tr}\left(\bar{J}_{1}(0)\right) \neq-2,-1,1,2$.

Now, the normal form is investigated. Let $\gamma=\mathcal{I} \Uparrow\left(\lambda_{1,2}\right), \delta=\operatorname{Re}\left(\lambda_{1,2}\right)$, and $T=\left[\begin{array}{ll}0 & 1 \\ \gamma & \delta\end{array}\right]$. Taking $\left[\begin{array}{l}u_{n} \\ v_{n}\end{array}\right]=T\left[\begin{array}{l}\bar{x}_{n} \\ \bar{y}_{n}\end{array}\right]$ to the model (7), we attain

$$
\begin{aligned}
& \bar{x}_{n+1}=\delta \bar{x}_{n}-\gamma \bar{y}_{n}+f_{1}\left(\bar{x}_{n}, \bar{y}_{n}\right) \\
& \bar{y}_{n+1}=\gamma \bar{x}_{n}+\delta \bar{y}_{n}+g_{1}\left(\bar{x}_{n}, \bar{y}_{n}\right)
\end{aligned}
$$

where the functions $f_{1}$ and $g_{1}$ denote the terms in the model (8) in variables $\left(\bar{x}_{n}, \bar{y}_{n}\right)$ with the order at least two.

In order to undergo Neimark-Sacker Bifurcation it requires that the following discriminatory quantity $\Omega$ be nonzero:

$$
\Omega=-\operatorname{Re}\left[\frac{(1-2 \bar{\lambda}) \bar{\lambda}^{2}}{1-\lambda} \xi_{11} \xi_{20}\right]-\frac{1}{2}\left|\xi_{11}\right|^{2}-\left|\xi_{02}\right|^{2}+\operatorname{Re}\left(\bar{\lambda} \xi_{21}\right)
$$

where

$$
\begin{aligned}
\xi_{20}= & \frac{1}{8} \delta(-4 b d-2 \delta b c+c+8 \gamma b c)-\frac{1}{4} \gamma c+\frac{b c}{2} \delta i(2 \gamma-1-\delta) \\
& +\frac{1}{2} i\left(-2 \gamma b d+\gamma^{2} b c+a\right)+\frac{1}{8} d+\frac{-2 \delta a}{4 \gamma}+\frac{2 b c \delta^{3}+2 b d \delta^{2}}{4 \gamma}-\frac{-c \delta^{2}-\delta d}{4 \gamma}, \\
\xi_{11}= & \frac{1}{2} \gamma(-2 b d-2 b c \delta)+\frac{1}{2} i\left(2 b c \gamma^{2}-2 a-c \delta+2 b c \delta^{2}\right)+\frac{\delta a}{\gamma} \\
& +\frac{\delta d+c \delta^{2}}{2 \gamma}-\frac{-2 \delta^{2} b d-2 b c \delta^{3}}{\gamma}, \\
\xi_{02}= & \frac{1}{4} \gamma(4 \delta b c-c-2 b d)+\frac{1}{4} i(d-4 \delta b d+2 c \delta+2 a)-\frac{2 \delta a+\delta d+c \delta^{2}}{4 \gamma} \\
& +\frac{1}{2} b c i\left(\gamma^{2}-3 \delta^{2}\right)-\frac{\delta^{2} b d+b c \delta^{3}}{2 \gamma} \\
\xi_{21}= & 0 .
\end{aligned}
$$

Finally, the following Theorem is achieved

Theorem 4. Let eq. (9) holds. When the parameter $b^{*}$ varies in a small neighborhood of $b$, the model (3) undergoes a Neimark-Sacker bifurcation around the fixed point $P_{2}=\left(x_{2}, y_{2}\right)$. If $\Omega<0$ (or $\Omega>0$ ) then an attracting (or repelling) invariant closed curve bifurcates from the fixed point 
$P_{2}=\left(x_{2}, y_{2}\right)$ for $b^{*}>0\left(\right.$ or $\left.b^{*}<0\right)$.

\subsection{Control Chaos}

To stabilize the chaotic orbits, the feedback control method is employed. The controlled form of model (3) is given by

$$
\begin{aligned}
& x_{n+1}=a x_{n}\left(1-x_{n}\right)-c\left(x_{n}-b y_{n}\right) y_{n}+S, \\
& y_{n+1}=d\left(x_{n}-b y_{n}\right) y_{n},
\end{aligned}
$$

with the feedback control law as the control force is $S=-q_{1}\left(x_{n}-x_{2}\right)-q_{2}\left(y_{n}-y_{2}\right)$, where $q_{1}$ and $q_{2}$ are the feedback gain. By computing the Jacobian Matrix of the model (10) at $\left(x_{2}, y_{2}\right)$, we have $J=\left[\begin{array}{cc}a_{11}-q_{1} & a_{12}-q_{2} \\ a_{21} & a_{22}\end{array}\right]$, where

$$
a_{11}=a(1-2 x)-c y, a_{12}=-c(x-2 b y), a_{21}=d y, a_{22}=d(x-2 b y),
$$

and the characteristic equation is

$$
\lambda^{2}-\left(a_{11}+a_{22}-q_{1}\right) \lambda+a_{22}\left(a_{11}-q_{1}\right)-a_{21}\left(a_{12}-q_{2}\right)=0 .
$$

Therefore we have the following eigenvalues properties.

$$
\lambda_{1}+\lambda_{2}=a_{11}+a_{22}-q_{1} \text { and } \lambda_{1} \lambda_{2}=a_{22}\left(a_{11}-q_{1}\right)-a_{21}\left(a_{12}-q_{2}\right)
$$

The lines of marginal stability are determined by solving the equation $\lambda_{1}= \pm 1$ and $\lambda_{1} \lambda_{2}=1$. These conditions guarantee that the eigenvalues $\lambda_{1}$ and $\lambda_{2}$ have modulus less than 1 .

Suppose $\lambda_{1} \lambda_{2}=1$; from (11), we have line $l_{1}$ as $a_{22} q_{1}-a_{21} q_{2}=a_{22} a_{11}-a_{21} a_{12}-1$.

Suppose $\lambda_{1}= \pm 1$; from (11), we have line $l_{2}$ and $l_{3}$ as follows:

$\left(1-a_{22}\right) q_{1}+a_{21} q_{2}=a_{11}+a_{22}-1-a_{22} a_{11}+a_{21} a_{12}$

and

$\left(1+a_{22}\right) q_{1}-a_{21} q_{2}=a_{11}+a_{22}+1+a_{22} a_{11}-a_{21} a_{12}$

The stable eigenvalues lie within a triangular region bounded by the lines $l_{1}, l_{2}$ and $l_{3}$.

Example: Let $a=4.42, b=0.08, c=3, d=3.5$. For initial value $\left(x_{0}, y_{0}\right)=(0.6,0.3)$, we have an unstable fixed point $P_{2}=(0.35,0.76)$ and nearby solution show the chaotic behavior, see Figure 1(a). Applying feedback control with feedback gain $q_{1}=0.2$ and $q_{2}=-0.5$, the stability of $P_{2}$ change sign as Figure 1(b).

\subsection{Numerical Simulations}

In this subsection, some numerical simulations are provided in order to depict the previous analytical results. The parameter values $a=3, b=0.08, c=3$, and $d=3.5$ are given hypothetically along with initial population $\left(x_{0}, y_{0}\right)=(0.6,0.3)$.

The bifurcation analysis of the proposed discrete predator-prey model with quantitative behavior has been presented to the parameter value. The bifurcation diagram is a numerical process that takes up a lot of time because it requires a humongous number 

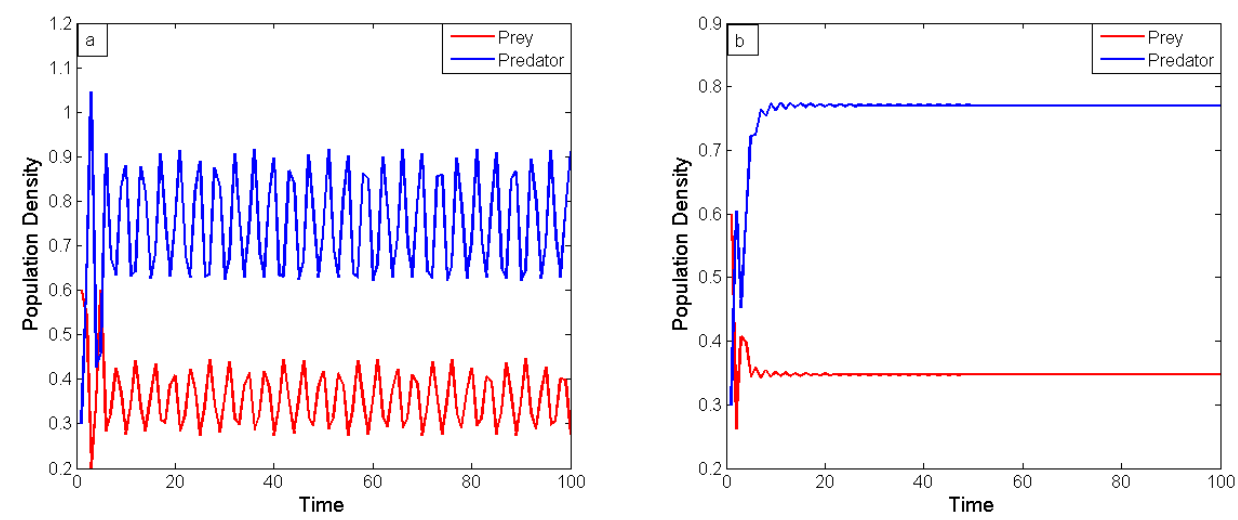

Figure 1. Chaos Control of discrete predator-prey model
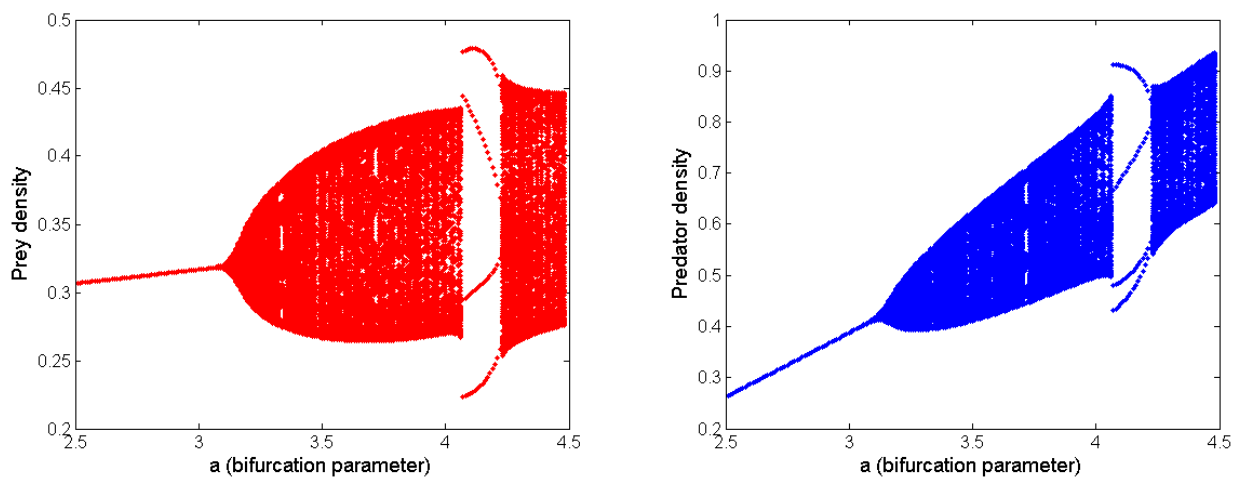

Figure 2. Bifurcation diagram driven by parameter $a$

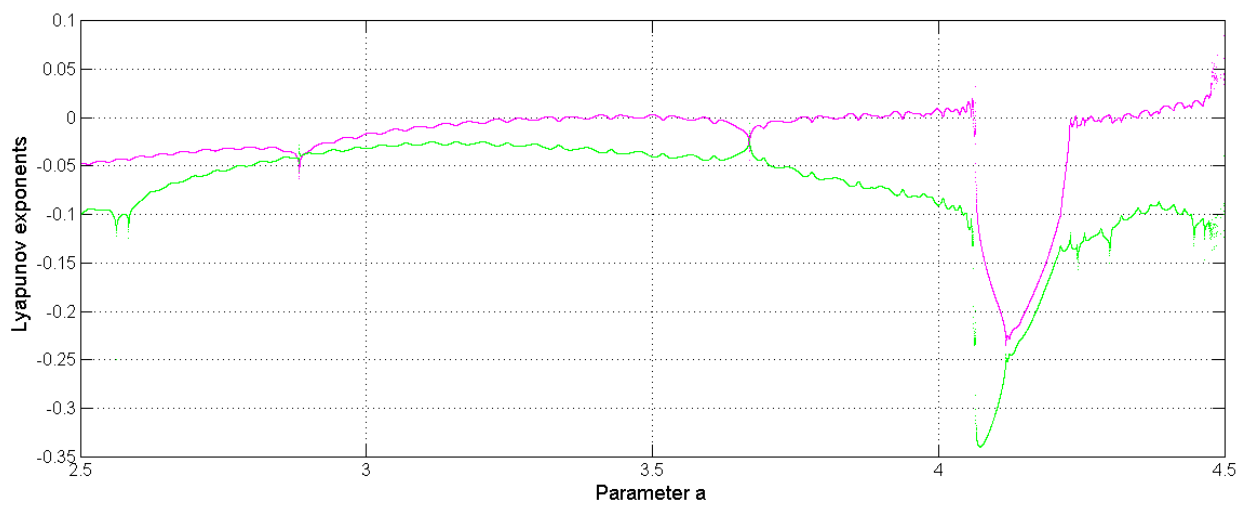

Figure 3. Lyapunov exponents by varying $a$

of points to describe the trajectory structure of the solution by varying the parameter. The Lyapunov exponents are also provided to investigate the rate of divergence of nearby trajectories as a key component of chaotic dynamics. The model with negative Lyapunov exponents is characteristic of dissipative or non-conservative, which are exhibited asymptotic stability. The resultant of more negative exponent implies the model's stability increase. The orbit is unstable and chaotic for positive Lyapunov.

By varying the parameter $a$ in the interval $[2.5,4.5]$, we obtain Figure 2 which depicts the existence of Neimark-Sacker bifurcation indicated by a change in behavior of the interior 

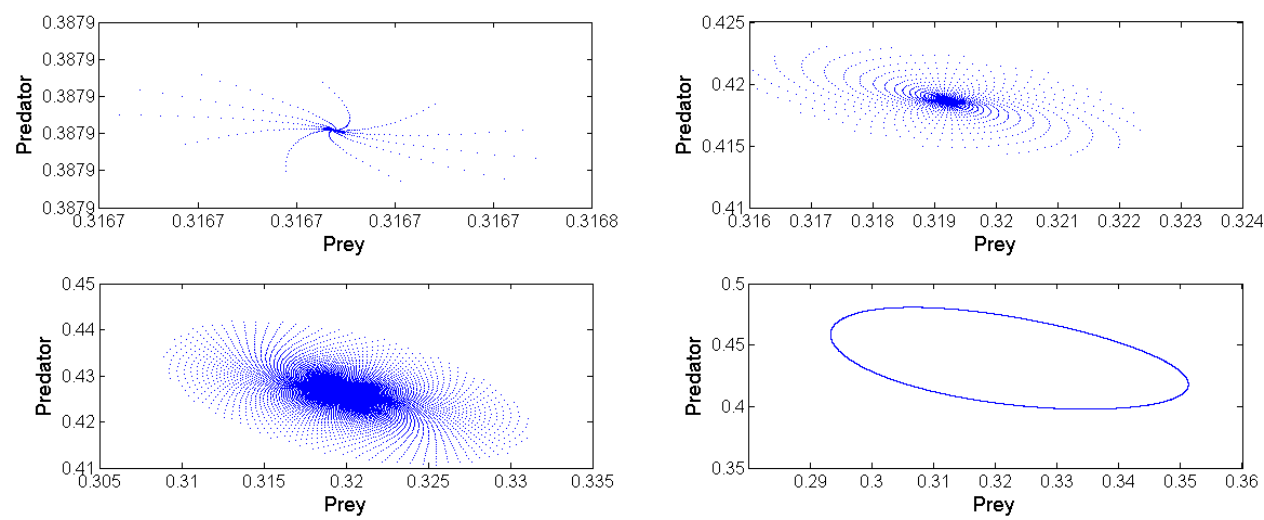

Figure 4. Phase portraits of the model for (i) $a=3$, (ii) $a=3.12$, (iii) $a=3.15$, (iv) $a=3.2$
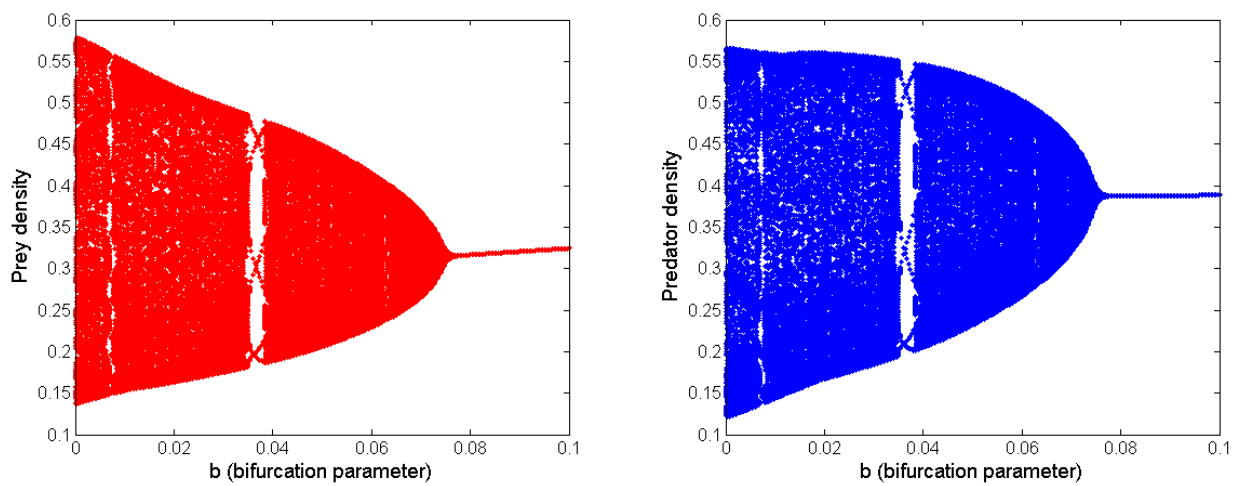

Figure 5. Bifurcation diagram driven by parameter $b$

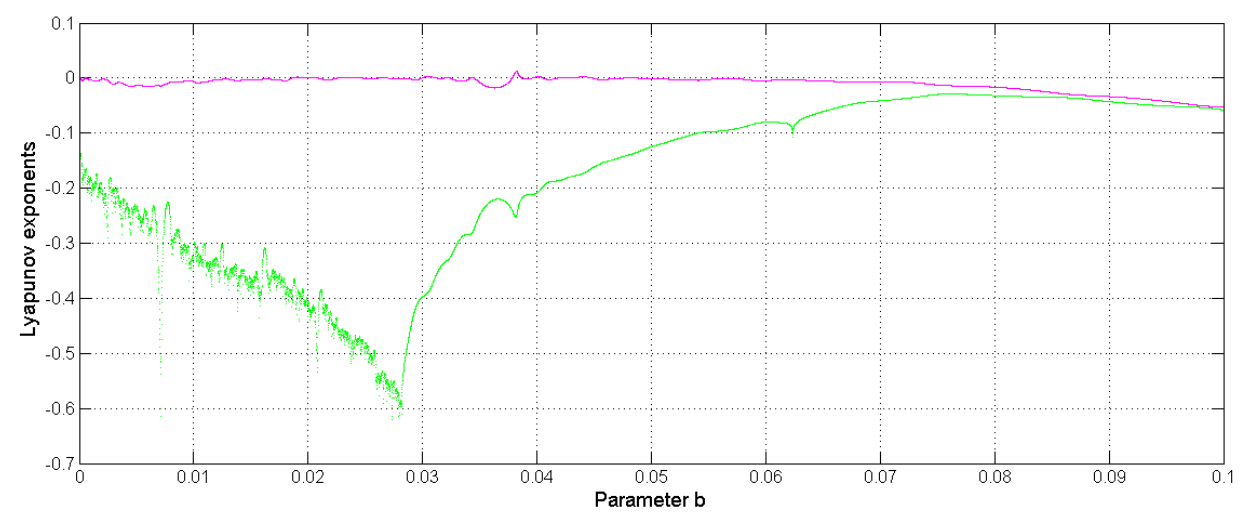

Figure 6. Lyapunov exponents by varying $b$

point and the occurrence of a smooth invariant circle. We also have a period-4 solution when $a>4$. To show the rate of the divergence, the appropriated Lyapunov exponent is given in Figure 3. The largest Lyapunov exponent is more significant than zero when $a>3.9$, except in periodic windows, which implies the occurrence of a chaotic solution. We set $a=3,3.12,3.15,3.2$ to describe some phase portrait as in Figure 4, which shows that the stable interior-point losses its stability, and the nearby solution tends to a smooth invariant closed curve. 

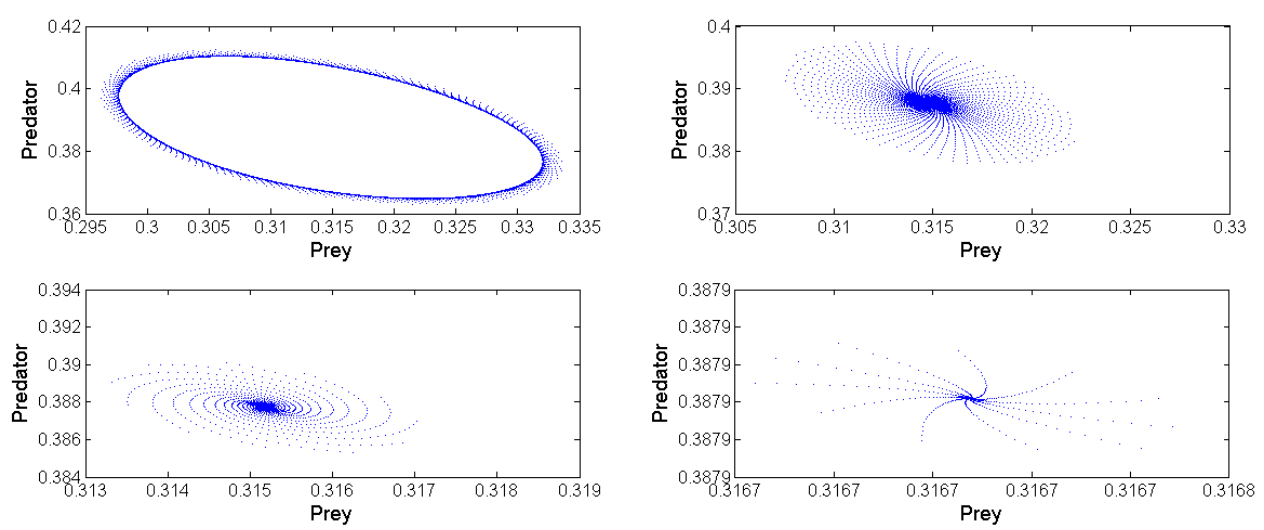

Figure 7. Phase portraits for $(i) b=0.074$, (ii) $b=0.075$, (iii) $b=0.076$, (iv) $b=0.08$
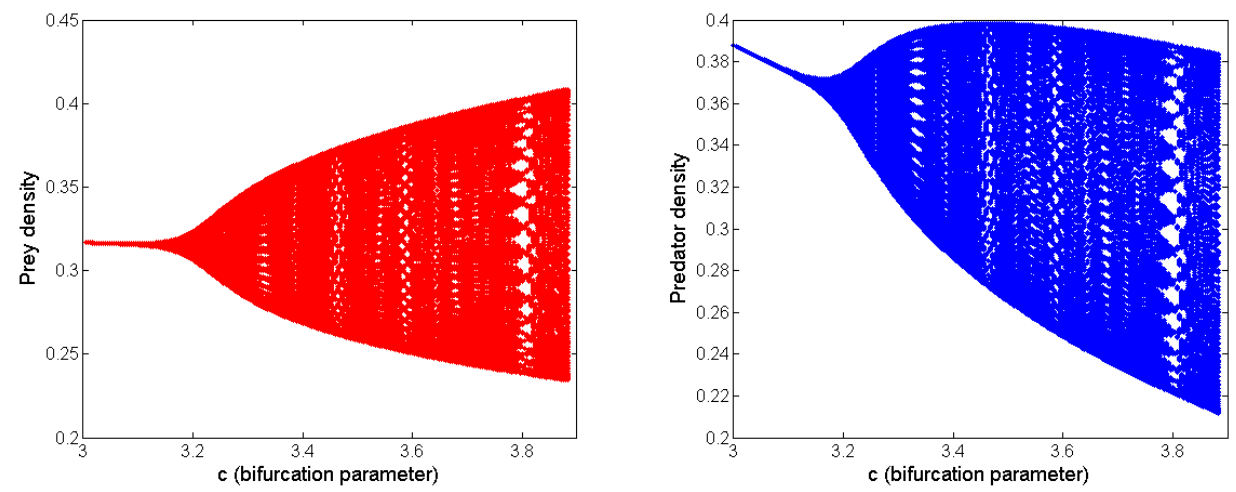

Figure 8. Bifurcation diagram driven by parameter $c$

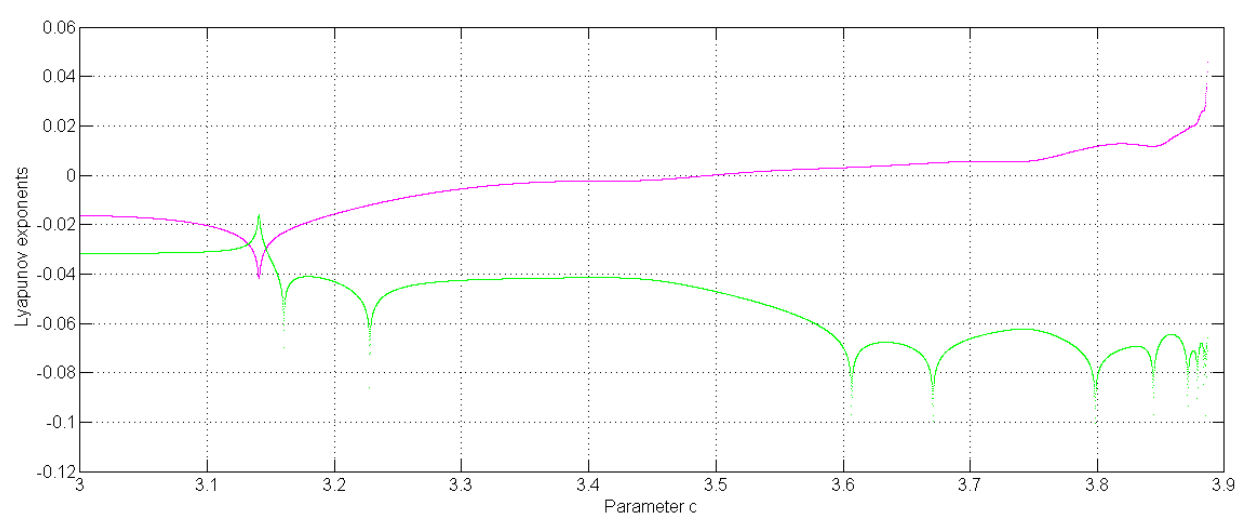

Figure 9. Lyapunov exponents by varying $c$

If we set parameter $b$ as the bifurcation parameter in interval $[0,0.1]$, we obtain the bifurcation diagram, Lyapunov exponent, and some phase portraits in Figures 5 to 7, respectively. The invariant circle which isolates the unstable interior-point vanishes and the interior point becomes stable via Neimark-Sacker bifurcation. The largest Lyapunov exponent indicates the chaotic behavior doesn't exist in this parameter interval. Four phase portraits are given to support this phenomenon. 

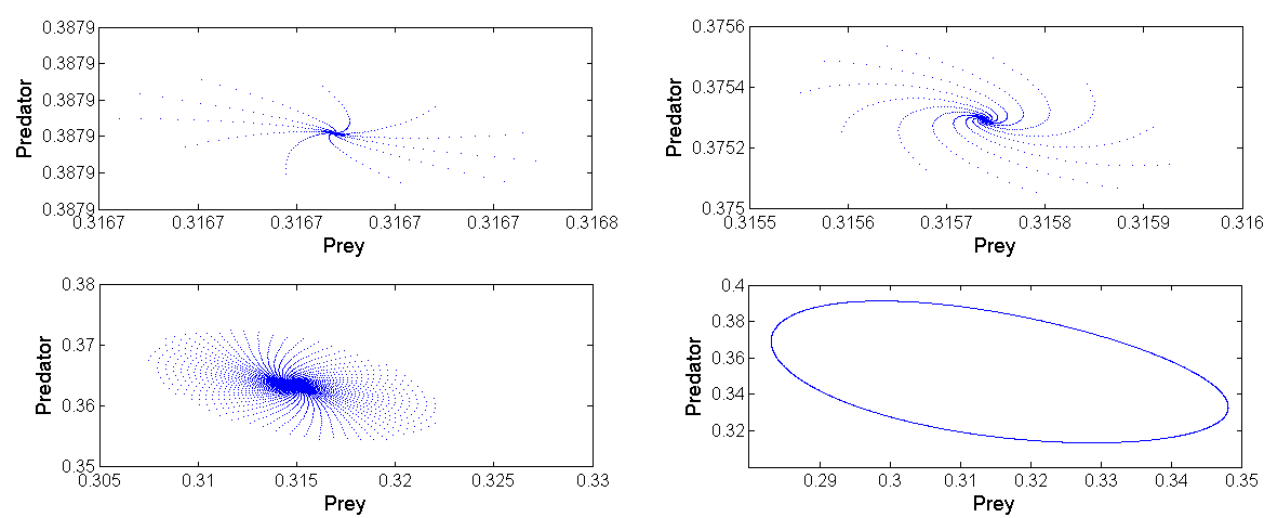

Figure 10. Phase portraits for $($ i) $c=3$, (ii) $c=3.1$, (iii) $c=3.2,(i v) c=3.3$
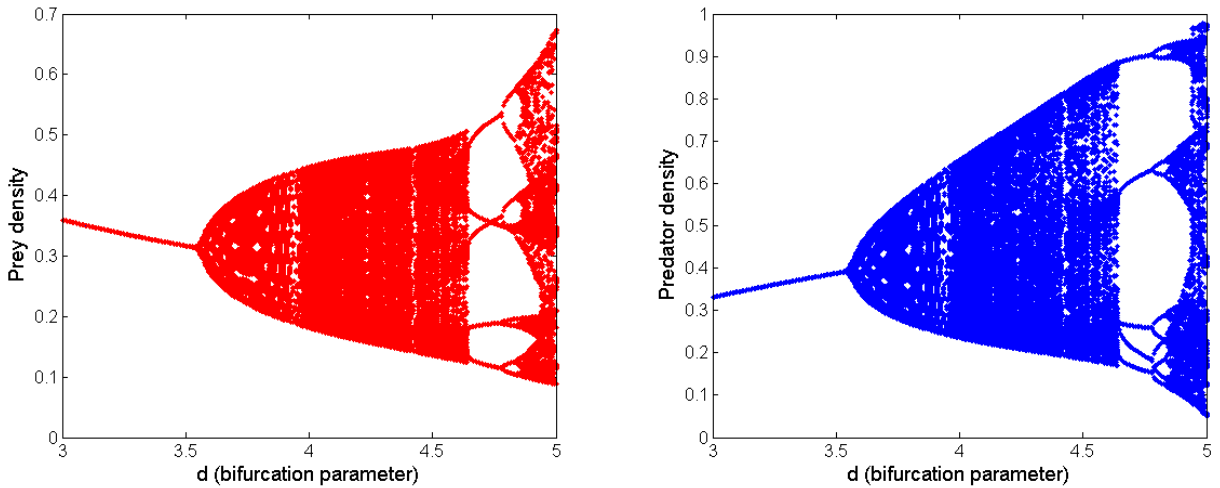

Figure 11. Bifurcation diagram driven by parameter $d$

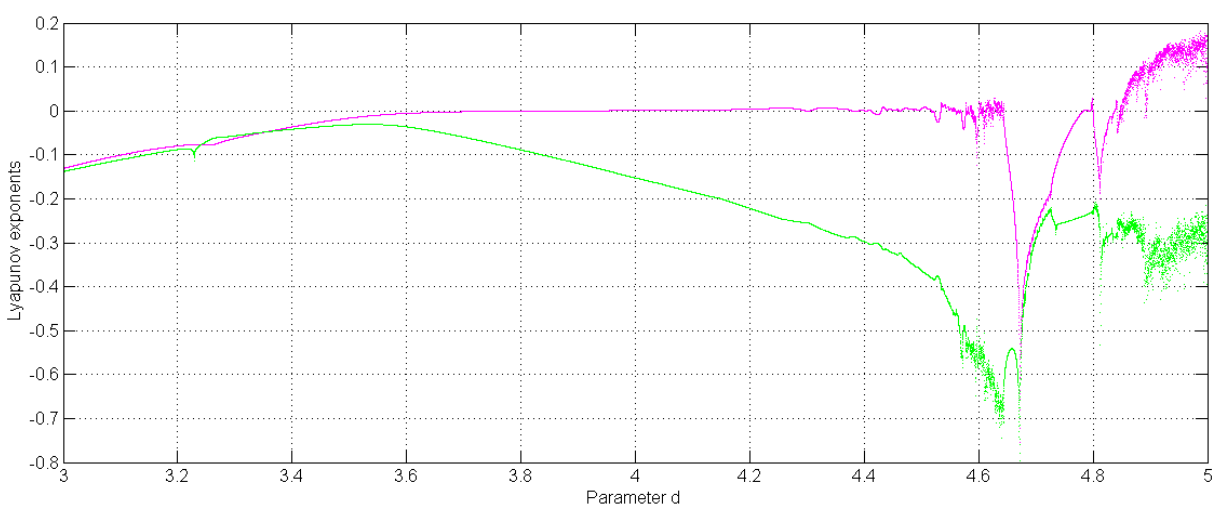

Figure 12. Lyapunov exponents by varying $d$

Now, we set $c$ as the bifurcation parameter. For $3 \leq c \leq 3.9$, the dynamical behaviors qualitative similar with the previous circumstance when $a$ is varied. The stable interiorpoint change sign and a smooth invariant circle appear simultaneously via NeimarkSacker bifurcation and finally, the chaotic attractor occurs. These conditions show by the bifurcation diagram Figure 8, the Lyapunov exponents Figure 9, and phase portraits Figure 10.

The last simulation is shown by setting $d$ as the bifurcation parameter. In interval $3 \leq$ 

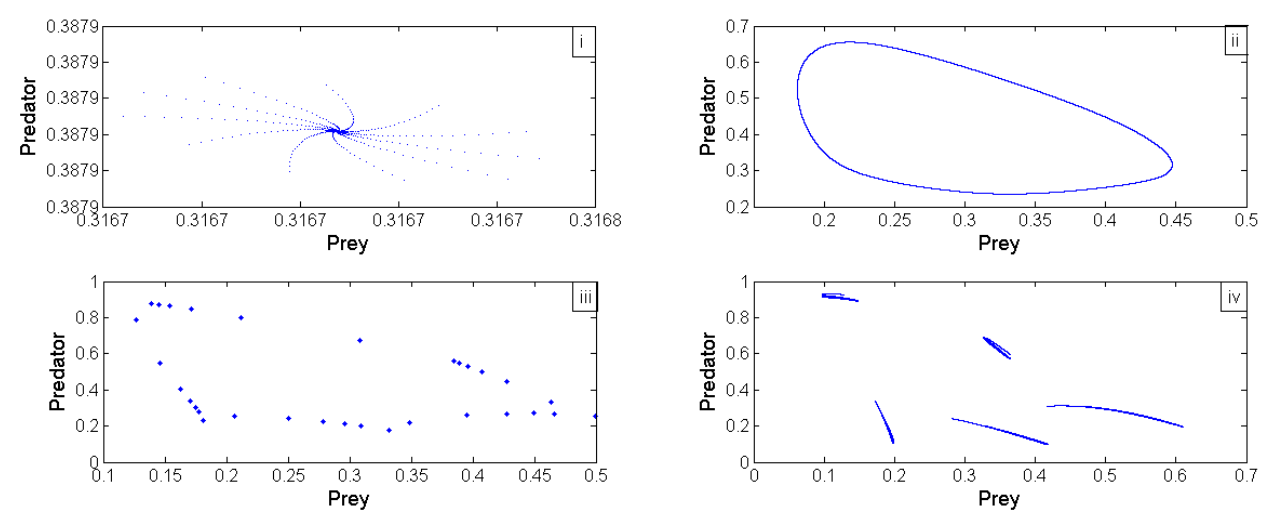

Figure 13. Phase portraits for $(i) d=3.5(i i) d=4.0(i i i) d=4.6(i v) d=4.9$

$d \leq 5$, we obtain bifurcation diagram, largest Lyapunov exponent, and phase portraits as in Figures 11 to 13. The Neimark-Sacker bifurcation occurs when $b$ passes through the bifurcation point approximately in $3.5<d<4$, and a big visible periodic window exists for $4.5<d<5$. Again, a chaotic solution appears indicated by the value of the Lyapunov exponent larger than zero, and a chaotic attractor is shown by Figure 13(d).

\section{Conclusion}

The dynamical behaviors of a predator-prey model with effects of refuge have been successfully studied in both analytical and graphical approaches. Three fixed points are investigated including their biological existence and their local stability conditions. The existence of Neimark-Sacker bifurcation is also investigated analytically by considering the effect of refuge as the bifurcation parameter. The feedback control method has been given to stabilize the chaotic solution. From a numerical approach, some results have been given. Some parameters in this model may affect the occurrence of Neimark-Sacker bifurcation, chaotic attractor, and some periodic solutions shown by the existence of periodic windows. This study of bifurcations in a discrete predator-prey model with refuge has been revealed that the model has complexity in dynamical behaviors shown by the existence of various bifurcations of codimension one, including flip bifurcations and Neimark-Sacker bifurcation, as the values of parameters vary. Generally, analytical and numerical results show that the refuge has impacts on predator density and changes the way of the predator and prey maintain their existence in nature.

\section{References}

[1] M. G. Neubert and M. Kot, "The subcritical collapse of predator populations in discretetime predator-prey models," Mathematical Biosciences, vol. 110, no. 1, pp. 45-66, 1992, doi: https://doi.org/10.1016/0025-5564(92)90014-N.

[2] J. Huang, S. Liu, S. Ruan, and D. Xiao, "Bifurcations in a discrete predator-prey model with nonmonotonic functional response," Journal of Mathematical Analysis and Applications, vol. 464, no. 1, pp. 201-230, 2018, doi: https://doi.org/10.1016/j.jmaa.2018.03.074.

[3] M. Gámez, I. López, C. Rodríguez, Z. Varga, and J. Garay, "Ecological monitoring in a discrete-time prey-predator model," Journal of Theoretical Biology, vol. 429, pp. 52-60, 2017, doi: https://doi.org/10.1016/j.jtbi.2017.06.025.

[4] A. Gkana and L. Zachilas, "Non-overlapping Generation Species: Complex Prey-Predator Interactions," International Journal of Nonlinear Sciences and Numerical Simulation, vol. 16, no. 5, pp. 207-219, 2015, doi: https://doi.org/10.1515/ijnsns-2014-0121. 
[5] E. Sebastian, A. Victor, Preethi Gkana, and L. Zachilas, "The dynamics of a discrete-time ratio-dependent prey-predator model incorporating prey refuge and harvesting on prey," International Journal of Applied Engineering Research, vol. 10, no. 55, pp. 2385-2388, 2015.

[6] P. K. Santra, G. S. Mahapatra, and G. R. Phaijoo, "Bifurcation and Chaos of a Discrete Predator-Prey Model with Crowley-Martin Functional Response Incorporating Proportional Prey Refuge," Mathematical Problems in Engineering, vol. 2020, pp. 1-18, 2020, doi: https: //doi.org/10.1155/2020/5309814.

[7] H. S. Panigoro, E. Rahmi, N. Achmad, S. L. Mahmud, R. Resmawan, and A. R. Nuha, "A discrete-time fractional-order Rosenzweig-Macarthur predator-prey model involving prey refuge," Communications in Mathematical Biology and Neuroscience, no. 1925, pp. 1-19, 2021, doi: https://doi.org/10.28919/cmbn/6586.

[8] P. Santra, "Discrete-time prey-predator model with $\theta$-logistic growth for prey incorporating square root functional response," Jambura Journal of Biomathematics, vol. 1, no. 2, pp. 41-48, 2020, doi: https://doi.org/10.34312/jjbm.v1i2.7660.

[9] P. K. Santra, "Fear effect in discrete prey-predator model incorporating square root functional response," Jambura Journal of Biomathematics, vol. 2, no. 2, pp. 51-57, 2021, doi: https://doi. org/10.34312/jjbm.v2i2.10444.

[10] P. Santra, G. S. Mahapatra, and D. Pal, "Prey-predator nonlinear harvesting model with functional response incorporating prey refuge," International Journal of Dynamics and Control, vol. 4, no. 3, pp. 293-302, 2016, doi: https://doi.org/10.1007/s40435-015-0198-6.

[11] Q. Wang, Z. Liu, X. Zhang, and R. A. Cheke, "Incorporating prey refuge into a predator-prey system with imprecise parameter estimates," Computational and Applied Mathematics, vol. 36, no. 2, pp. 1067-1084, 2017, doi: https://doi.org/10.1007/s40314-015-0282-8.

[12] C. Banerjee and P. Das, "Impulsive Effect on Tri-Trophic Food Chain Model with Mixed Functional Responses under Seasonal Perturbations," Differential Equations and Dynamical Systems, vol. 26, no. 1-3, pp. 157-176, 2018, doi: https:/ / doi.org/10.1007/s12591-016-0328-4.

[13] B. Sahoo and S. Poria, "Oscillatory Coexistence of Species in a Food Chain Model With General Holling Interactions," Differential Equations and Dynamical Systems, vol. 22, no. 3, pp. 221-238, 2014, doi: https://doi.org/10.1007/s12591-013-0171-9.

[14] H. S. Panigoro, A. Suryanto, W. M. Kusumahwinahyu, and I. Darti, "Dynamics of a fractional-order predator-prey model with infectious diseases in prey," Communication in Biomathematical Sciences, vol. 2, no. 2, p. 105, 2019, doi: https://doi.org/10.5614/cbms.2019. 2.2.4.

[15] H. S. Panigoro, A. Suryanto, W. M. Kusumawinahyu, and I. Darti, "Dynamics of an eco-epidemic predator-prey model involving fractional derivatives with power-law and mittag-leffler kernel," Symmetry, vol. 13, no. 5, p. 785, 2021, doi: https://doi.org/10.3390/ sym13050785.

[16] L. K. Beay and M. Saija, "A stage-structure Rosenzweig-MacArthur model with effect of prey refuge," Jambura Journal of Biomathematics, vol. 1, no. 1, pp. 1-7, 2020, doi: https://doi.org/ 10.34312/jjbm.v1i1.6891.

[17] Y. Wu, F. Chen, and C. Du, "Dynamic behaviors of a nonautonomous predator-prey system with Holling type II schemes and a prey refuge," Advances in Difference Equations, vol. 2021, no. 1, pp. 1-15, 2021, doi: https://doi.org/10.1186/s13662-021-03222-1.

[18] Y. Tao, X. Wang, and X. Song, "Effect of prey refuge on a harvested predator-prey model with generalized functional response," Communications in Nonlinear Science and Numerical Simulation, vol. 16, no. 2, pp. 1052-1059, 2011, doi: https://doi.org/10.1016/j.cnsns.2010.05. 026.

[19] H. S. Panigoro, E. Rahmi, N. Achmad, and S. L. Mahmud, "The influence of additive Allee effect and periodic harvesting to the dynamics of Leslie-Gower predator-prey model," Jambura Journal of Mathematics, vol. 2, no. 2, pp. 87-96, 2020, doi: https://doi.org/10.34312/ jjom.v2i2.4566.

[20] H. S. Panigoro, A. Suryanto, W. M. Kusumawinahyu, and I. Darti, "Global stability of a fractional-order Gause-type predator-prey model with threshold harvesting policy in predator," Communications in Mathematical Biology and Neuroscience, vol. 2021, no. 2021, p. 63, 2021, doi: https://doi.org/10.28919/cmbn/6118. 
[21] G. Ruxton, "Short Term Refuge Use and Stability of Predator-Prey Models," Theoretical Population Biology, vol. 47, no. 1, pp. 1-17, 1995, doi: https://doi.org/10.1006/tpbi.1995.1001.

[22] R. Cressman and J. Garay, "A predator-prey refuge system: Evolutionary stability in ecological systems," Theoretical Population Biology, vol. 76, no. 4, pp. 248-257, 2009, doi: https://doi.org/10.1016/j.tpb.2009.08.005.

[23] U. Ufuktepe, B. Kulahcioglu, and O. Akman, "Stability analysis of a prey refuge predator-prey model with Allee effects," Journal of Biosciences, vol. 44, no. 4, p. 85, 2019, doi: https://doi.org/10.1007/s12038-019-9911-5.

[24] S. Kapçak, S. Elaydi, and Ü. Ufuktepe, "Stability of a predator-prey model with refuge effect," Journal of Difference Equations and Applications, vol. 22, no. 7, pp. 989-1004, 2016, doi: https://doi.org/10.1080/10236198.2016.1170823.

[25] A. Gkana and L. Zachilas, "Incorporating prey refuge in a prey-predator model with a Holling type I functional response: random dynamics and population outbreaks," Journal of Biological Physics, vol. 39, no. 4, pp. 587-606, 2013, doi: https://doi.org/10.1007/ s10867-013-9319-7.

[26] F. Chen, L. Chen, and X. Xie, "On a Leslie-Gower predator-prey model incorporating a prey refuge," Nonlinear Analysis: Real World Applications, vol. 10, no. 5, pp. 2905-2908, 2009, doi: https://doi.org/10.1016/j.nonrwa.2008.09.009.

[27] Z. Ma, S. Wang, W. Li, and Z. Li, "The effect of prey refuge in a patchy predator-prey system," Mathematical Biosciences, vol. 243, no. 1, pp. 126-130, 2013, doi: https://doi.org/ 10.1016/j.mbs.2013.02.011.

[28] G. S. Mahapatra and P. Santra, "Prey-predator model for optimal harvesting with functional response incorporating prey refuge," International Journal of Biomathematics, vol. 09, no. 01, p. 1650014, 2016, doi: https://doi.org/10.1142/S1793524516500145.

[29] R. N. Fan, "A Predator-Prey Model Incorporating Prey Refuge and Allee Effect," Applied Mechanics and Materials, vol. 713-715, pp. 1534-1539, 2015, doi: https://doi.org/10.4028/ www.scientific.net/AMM.713-715.1534.

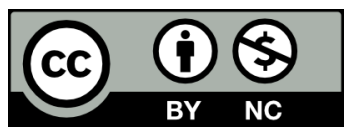

This article is an open-access article distributed under the terms and conditions of the Creative Commons Attribution-NonCommercial 4.0 International License. Editorial of JJoM: Department of Mathematics, Universitas Negeri Gorontalo, Jln. Prof. Dr. Ing. B.J. Habibie, Moutong, Tilongkabila, Kabupaten Bone Bolango, Provinsi Gorontalo 96119, Indonesia. 